Abstract: Recent results from high intensity (up to $5 \times 10^{20} \mathrm{~W} / \mathrm{cm}^{2}$ ) laser plasma interaction experiments at Imperial College London have shown that the plasmas produced during such interactions can be efficient sources of relativistic electron beams and also of high quality beams of non-relativistic ions. These beams may be important for the development of compact sources of energetic particles for applications in science, medicine and technology.

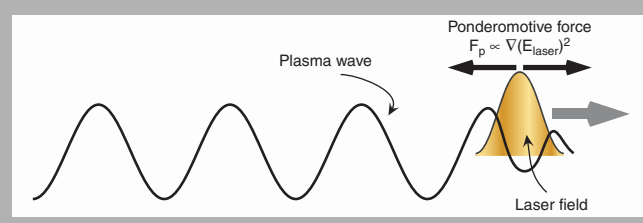

Schematic of laser wakefield acceleration

\title{
Particle acceleration using intense laser produced plasmas
}

\author{
K. Krushelnick, ${ }^{1,2, *}$ Z. Najmudin, ${ }^{1}$ and A.E. Dangor ${ }^{1}$ \\ ${ }^{1}$ Department of Physics, Imperial College London, SW7 2AZ, UK \\ ${ }^{2}$ Center for Ultrafast Optical Science (CUOS), University of Michigan, Ann Arbor 48109, USA
}

Received: 6 June 2007, Revised: 9 July 2007, Accepted: 15 July 2007

Published online: 3 August 2007

Key words: laser plasma interaction; electron acceleration; ion acceleration

PACS: $52.38-\mathrm{r}, 52-38 . \mathrm{Kd}$

\section{Introduction}

Since the first electron cyclotron was built by E.O. Lawrence at Berkeley in the 1930's, the technology of particle acceleration has developed considerably. Indeed, the peak energy of electrons accelerated by these devices has risen from several Megaelectronvolts (MeV) during the first experiments to around 100 Gigaelectronvolts (GeV) in the 1980's and 90's using the Large Electron Positron Collider (LEP) at CERN. This energy increase of over five orders of magnitude has enabled significant advances in the understanding of the nature of matter and also of the fundamental forces. The next large electron accelerator is planned to be the International Linear Collider (ILC) - designed to accelerate electrons to $\mathrm{TeV}$ energies (another order of magnitude increase) - and will have an acceleration distance of tens of kilometers. The cost of such systems is enormous and there is a considerable research effort around the world to explore new technological possibilities for achieving energies even beyond this.

Particle accelerators have also been developed for a vast variety of other applications, which are important in science, medicine and technology and which have a much more direct effect on our everyday lives. For example, small-scale accelerators are used to produce x-rays to characterize material properties as well as to generate nuclear isotopes for medical applications.

One of the limitations for conventional accelerator technology is that if the radiofrequency electric fields used to accelerate the particles are too large, the material in the walls of the accelerator can "break down" or become ionized (i.e., it forms a plasma) - this consequently changes the structure of the accelerating field and destroys the uniformity of the accelerated electron beam. This limitation in maximum accelerating field is fundamentally why accelerators generally need to be very large - because, for example, to get to $\mathrm{TeV}$ energies with an electric field low enough to prevent breakdown, it is necessary to accelerate the particle over a distance of many kilometers.

One idea to go beyond such limitations is to use plasma as the accelerating medium. Much higher electric fields are possible in a plasma than in an unionized material so there is the possibility of using plasmas as "compact" high field accelerators. The main problem with this approach is

\footnotetext{
* Corresponding author: e-mail: kmkr@umich.edu
} 


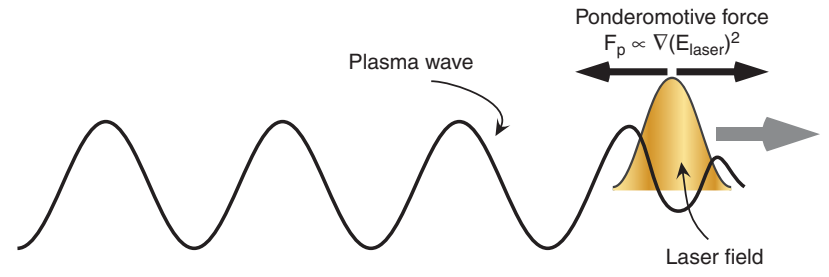

Figure 1 (online color at www.lphys.org) Schematic of laser wakefield acceleration

that plasmas are notoriously difficult to control since they are affected by a host of instabilities. Understanding how to control plasmas so that they can be incorporated effectively into technology remains a significant challenge.

In this brief review paper we will discuss recent research conducted at Imperial College London over the past several years towards the development of laser based "compact" plasma accelerators for both electrons and ions.

\section{Electron acceleration using high intensity lasers}

Since the invention of the laser more than 40 years ago the power of laser beams has increased very rapidly. This has been mainly achieved mainly by reducing the duration of the pulse produced from the laser system. In fact, lasers today are capable of emitting pulses on the order of several femtoseconds $\left(10^{-15} \mathrm{~s}\right)$. Such short pulse high power lasers can consequently be focused to very high intensities. At intensities greater than about $10^{15} \mathrm{~W} / \mathrm{cm}^{2}$ the laser field immediately rips off the electrons from atoms - forming a plasma. The technology of these high power lasers has advanced significantly over the past several years and the peak intensity of laser systems has risen even more rapidly than the peak energies produced by electron accelerators.

In 1979, it was realised by Toshi Tajima and John Dawson of UCLA that high intensity laser pulses will also efficiently produce relativistic electron plasma waves as they travel through a low density plasma [1]. Electron plasma waves can be thought of as simply oscillating displacements of free electrons in the plasma from the neutralizing background of slower moving positively charged ions. These displacements of electrons give rise to large electric fields - which can be much larger than any fields possible in a non-ionized material. Tajima and Dawson realised that by using the light pressure of a focused laser pulse such waves could be generated in the wake of a very short laser pulse as it travelled though the plasma. This large amplitude electric field travelling at the speed of light is exactly what is required for efficient acceleration of relativistic particles - and consequently they proposed that plasma waves created in this way could be used for very high gradient particle accelerators (see Fig. 1). In this situation the electrons can "surf" on the plasma wave picking up energy from the wave just as a surfer picks up energy from a water wave in the ocean.

When they wrote their paper - the lasers necessary to produce these accelerating structures did not exist. However, now the technology to produce such intensities is indeed commonplace at major research universities and at national laboratories. In fact, it is presently possible to obtain focused intensities greater than $10^{20} \mathrm{~W} / \mathrm{cm}^{2}$ using laser systems, which have reasonably high repetition rates and which can fit into a university scale lab. These lasers are also capable of producing plasmas with very unusual properties - for example, they can have relativistic "temperatures" (the average energy in the system is higher than the electrons rest mass) [2] as well as ultra-strong (Gigagauss) magnetic fields [3].

There have been many experiments in the past several years, which have demonstrated that energetic electron beams can be produced as a result of the interaction of high intensity laser pulses with low density plasmas [420]. Relativistic electrons can be generated through the wavebreaking of the large amplitude relativistic plasma waves, which are created in the wake of the laser pulse as it propagates through the plasma and also through a direct interaction between the laser field and the electrons in the plasma. Electrons having an energy up to $350 \mathrm{MeV}$ have been observed from laser plasma interactions using PetaWatt-scale $\left(10^{15} \mathrm{~W}\right)$ lasers such that the laser field itself plays a major role in the acceleration process [9]. Such energies were obtained from acceleration distances of a millimeter or less. Although the relativistic electron bunches generated from these experiments are highly directional and contain high charge, they are emitted with an extremely large energy spread, which makes many of the potentially important applications for electron beams unfeasible with these sources.

Recently it has been shown that in high power experiments using much shorter pulses (in the ten's of femtoseconds regime), it is possible to generate true "beams" of relativistic electrons, which have low divergence and which have a relatively small energy spread $(<5 \%)$ [7,8,21]. And using an extended interaction length (a plasma waveguide formed from capillary discharge plasma of $3 \mathrm{~cm}$ in length) it has been shown that such beams can be produced up to $1 \mathrm{GeV}$ in energy [22].

This is an extremely important result since only if narrow energy bandwidths are achievable will the full range of applications become possible. The use of plasma acceleration consequently now offers the potential of significantly smaller and cheaper facilities for generating energetic electron beams, which, considered along with the current rapid developments in laser technology, could soon allow the construction of university laboratory sized accelerators for use in a wide range of experiments and applications. For example, table-top narrowband femtosecond $\mathrm{x}$-ray sources and free-electron lasers could become a reality - which may potentially lead to significant advances in both medicine and materials science. It may also be possible to use electron bunches generated in this way for in- 


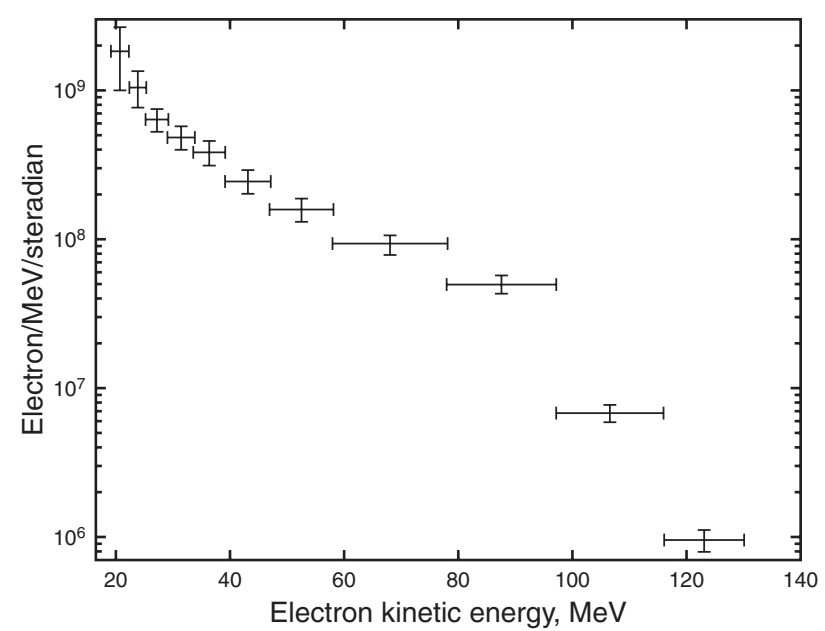

Figure 2 Typical electron spectrum from self-modulated laser wake field from a $50 \mathrm{TW}$ laser interaction with a helium gas jet target $\left(n_{e}=3 \times 10^{19} \mathrm{~cm}^{-3}\right)$

jection into conventional rf accelerators or into subsequent plasma acceleration stages [23].

\subsection{Self-modulated laser wakefield acceleration}

High field electron acceleration techniques have typically relied on electron plasma waves as the accelerating medium. In the laser wakefield accelerator concept [1], these waves are excited immediately behind the laser pulse as it propagates through the plasma. Efficient energy transfer between the plasma wave and the electrons requires that both move at similar velocities and this is achieved through the use of low density plasmas (less than $10^{20} \mathrm{~cm}^{-3}$ ), in which the phase velocity $v_{\phi}$ of the laserexcited plasma wave is equal to the laser pulse group velocity (which is close to the speed of light in vacuum, $c$ ). The longitudinal electric fields associated with the relativistic plasma waves are then able to accelerate relativistic particles injected externally, or even, for large amplitude waves, to trap particles from the plasma itself. Subsequently, particles can be boosted to high energy over very short distances by "surfing" on this electrostatic wave.

Acceleration schemes using lasers use the ponderomotive force of either a single very short pulse or a train of very short light pulses, each tailored to resonantly drive the relativistic plasma wave (resonance occurs when the laser pulse duration is about half of the electron plasma period, $T_{p}$ ).

Thus far, the schemes studied for producing wakefields are the laser wake field accelerator (LWFA) [1], the laser beat wave accelerator (LBWA) [23-26], the selfmodulated laser wake field accelerator (SM-LWFA) $[4,6]$, and the forced laser wake field (F-LWFA) [5,18] accelerator. In the LWFA the laser pulse pushes electrons at its leading and trailing edges with optimal coupling when the resonance condition mentioned above is satisfied. Electrons injected at $3 \mathrm{MeV}$ have been accelerated by this scheme up to $4.7 \mathrm{MeV}$ [20]. In the LBWA, a train of short pulses (a beat frequency) is produced by co-propagating two laser pulses at slightly different wavelengths. Electric fields close to the $\mathrm{GV} / \mathrm{m}$ level have been measured for this experimental configuration using $\mathrm{CO}_{2}$ laser beams and injected electrons have been accelerated up to $30 \mathrm{MeV}$ [24]. In LBWA, only electrons injected externally into the wave have been accelerated. In contrast, in the SM-LWFA and F-LWFA and resonant LWFA schemes, the plasma wave amplitude becomes so large that electrons from the plasma itself are trapped by the wave and are boosted to very high energies (i.e., the plasma wave breaks so that no external electron source is needed).

The SM-LWFA regime uses long $\left(\tau_{L} \gg T_{p}\right.$, where $\tau_{L}$ is the laser pulse duration) laser pulses at intensities sufficient to strongly excite the self-modulation instability, which is related to stimulated forward Raman scattering. In this process, the laser pulse self-focuses and scatters upon encountering electron plasma waves. The beating of the scattered electromagnetic wave with the laser light amplifies the plasma waves, leading to instability and plasma wave growth. As a result, the initial laser pulse is strongly modulated and is gradually transformed into a train of very short pulses that naturally satisfies the resonance condition. The plasma can also act as a converging lens due to relativistic effects and can focus the laser beam, permitting interactions at higher intensity than in vacuum, and over a longer distance than the natural diffraction length.

An example of an electron spectrum from a SM-LWFA experiment is shown in Fig. 2 [6]. This was produced from a laser plasma interaction experiment using the Vulcan laser system at the Rutherford Appleton Laboratory in the UK operating at $50 \mathrm{TW}$. This interaction was performed at a plasma density of $\sim 3 \times 10^{19} \mathrm{~cm}^{-3}$, a laser intensity of $\sim 10^{19} \mathrm{Wcm}^{-2}$ and a pulse length of about $1 \mathrm{ps}$. In this regime the peak electron energy observed was about $120 \mathrm{MeV}$ - although because the acceleration mechanism is caused by an instability, experiments in this regime are characterized by considerable shot-to-shot fluctuations in the peak electron energy and in the total accelerated charge. Typical forward scattered laser spectra are shown in Fig. 3. These show the characteristic scattered antiStokes sidebands (separated by the electron plasma frequency), which can be correlated to the production of the relativistic electron beam. The behavior of the sidebands on the Stokes (downshifted) side of the laser frequency is similar. As the plasma density increases the number of accelerated electrons can also dramatically increase - due to wavebreaking of the waves and self-trapping of electrons in the wake. This is evident in the forward scattered spectrum as a distinct broadening of the sidebands due to the transition from scattering from a large amplitude "single frequency" plasma wave to a scattering from a non-linear "broken" wave. 

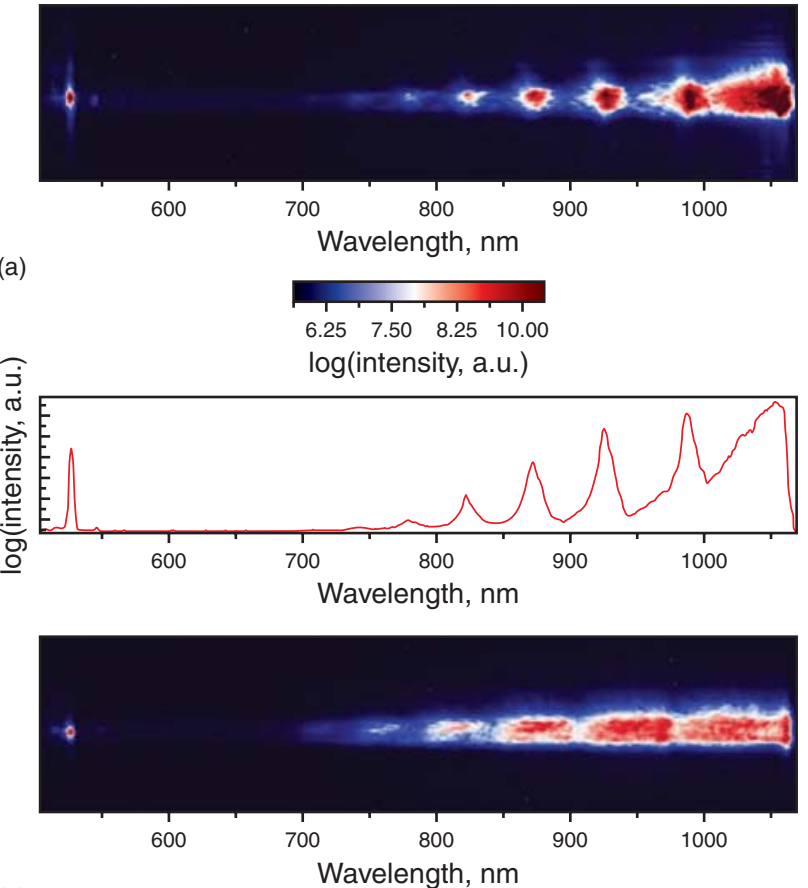

(b)

Wavelength, nm

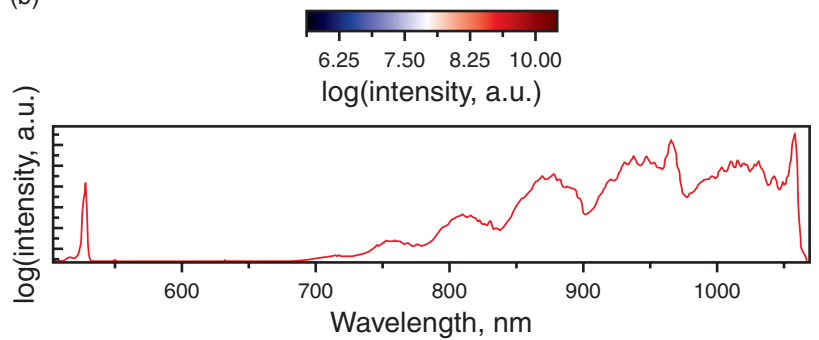

Figure 3 (online color at www.lphys.org) Transmitted beam spectra with (a) $n_{e}=4 \times 10^{18} \mathrm{~cm}^{-3}$ and $I=2 \times 10^{19} \mathrm{Wcm}^{-2}$ and (b) $n_{e}=7.5 \times 10^{18} \mathrm{~cm}^{-3}$ and $I=1 \times 10^{19} \mathrm{Wcm}^{-2}$. The signal at $527 \mathrm{~nm}$ is due to the second harmonic. Stokes satellites (down shifted satellites) are not shown because the CCD detector used is not sensitive at those wavelengths. The original raw data is displayed at the top of both (a) and (b) and a corrected lineout of the data is shown below

The divergence of the high-energy electrons from interactions in the SM-LWFA typically increases with density and can reach values up to 15 degrees for electrons greater than $8 \mathrm{MeV}$ [6].

\subsection{Laser acceleration of electrons at intensities greater than $10^{20} \mathrm{~W} / \mathrm{cm}^{2}$}

The recent development of Petawatt class lasers such as that at the Central Laser Facility at the Rutherford Appleton Laboratory in the UK [2] has allowed experiments to be performed at much higher intensities than previously available. The intensity of a laser system is often

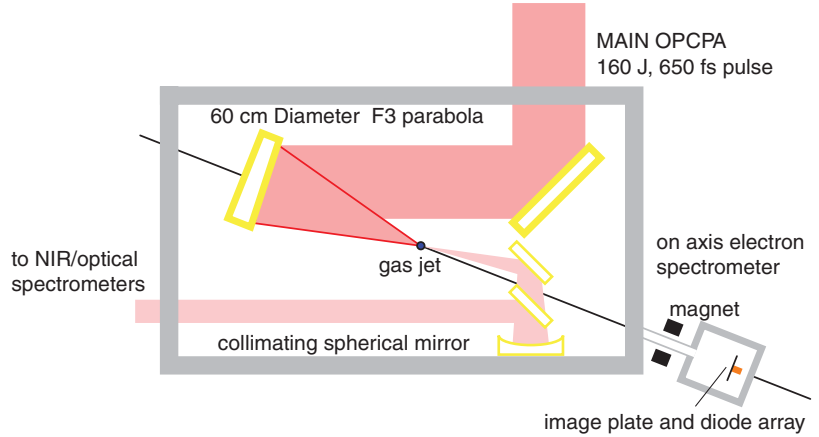

Figure 4 (online color at www.lphys.org) Schematic of experimental setup for Petawatt electron acceleration experiments

described by the normalised vector potential of the laser field, $a=e A / m_{e} c$ ( $A$ is the vector potential of the laser field and $m_{e}$ is the electron mass). $a$ is also the normalised transverse momentum of the electron motion in the laser field. As the time-averaged vector potential $a_{0}$ approaches 1 the electron motion becomes relativistic. The previous experiments as described up until now were performed with $a_{0}$ between 1 and 5. The Vulcan Petawatt facility allows experimental access to regimes where $a_{0} \gg 1$ (and in the experiment reported here $a_{0} \approx 15$ ).

During this experiment [9] the laser consistently produced 650 fs duration pulses delivering $\sim 180 \mathrm{~J}$ on target. These pulses were focused with an $f / 3$ off-axis parabolic mirror to produce a focal spot with an intensity FWHM (full width half maximum) of $\sim 10 \mu \mathrm{m}$, thus generating intensities greater than $3 \times 10^{20} \mathrm{Wcm}^{-2}$.

The experimental setup is shown in Fig. 4. The gas jet used had a $2 \mathrm{~mm}$ diameter supersonic nozzle and could produce plasma electron densities between $5 \times 10^{18}$ and $2 \times 10^{20} \mathrm{~cm}^{-3}$. The density was controlled by varying the backing pressure of helium behind the valve of the gas jet and can be measured using the forward Raman scattering signal.

The electrons accelerated along the axis of laser propagation were measured using a high field magnetic spectrometer. The electrons exit the highly shielded main vacuum chamber through a small $(25 \mathrm{~mm})$ diameter tube to a secondary vacuum vessel - and this helps to reduce the level of background signal from low energy $\mathrm{x}$-rays and scattered electrons. The entrance to the spectrometer is a $5 \mathrm{~mm}$ diameter hole that serves to collimate the electron beam to ensure sufficient energy resolution. The specially designed vacuum chamber allows the electron beam to pass between the pole pieces of an electromagnet that deflects the electrons off-axis. The correspondence between electron energy and deflection from the axis is determined by using a charged particle tracking code.

In these experiments, electrons are detected using an image plate, (Fuji BAS1800II) which is a re-usable film sensitive to ionizing radiation. Two sections of image plate 


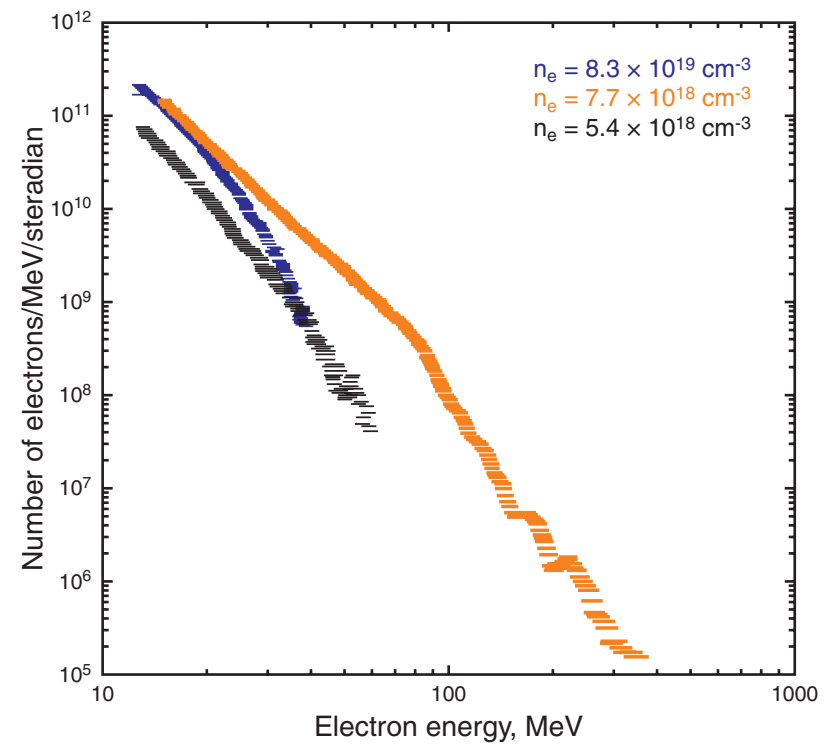

Figure 5 (online color at www.lphys.org) Electron energy spectra at three densities from Petawatt interactions. The highest energy electrons are observed at an electron density of $n=(7.7 \pm 0.7) \times 10^{18} \mathrm{~cm}^{-3}$. At this density the spectrum is non-Maxwellian. For densities higher or lower than this the spectra are broadly similar and can be better described by an effective temperature

are used, one to measure the electron signal, below axis, and another to measure the background signal above the axis. Since the background is due to $\mathrm{x}$-rays from the interaction itself or from bremsstrahlung radiation emitted by the electrons as they pass through material before the detector plane it is assumed that the background is symmetric above and below the axis.

The relationship between image plate signal intensity and energy deposited in the plate is close to linear. The direct relationship between the number of electrons and the signal was calculated by placing a diode array directly behind the image plates. The ion implanted diodes used have an absolutely calibrated response to the number of electrons incident and by calculating the energy lost by the electrons as they travel through the plate before reaching the diodes it is possible to cross-calibrate the diode and image plate signals.

The image plate data exhibits a much larger dynamic range than the diodes and the image nature of the data allows much better noise discrimination. The resolution of the image plates, although not as high as X-ray film is significantly better than the diode array. The combination of the resolution and size of the image plates (each is $250 \mathrm{~mm}$ long) allows a reasonably broad energy range (for example $10-250 \mathrm{MeV}$ ) to be measured in a single shot.

The laser-plasma interaction was also diagnosed by measuring the transmitted spectrum of the laser. A portion of the transmitted beam was collimated and transported

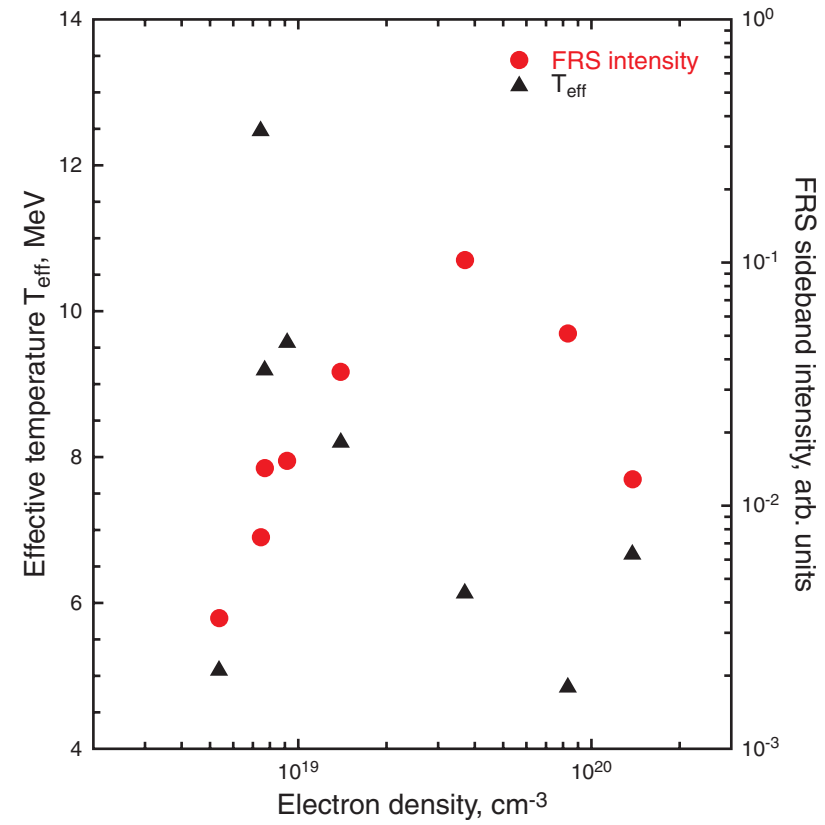

Figure 6 (online color at www.lphys.org) Variation of observed electron "temperature" and amount of forward Raman scattered (FRS) light with plasma density in helium gas jet. Note the similar behavior in both data sets and the lack of correlation of FRS and acceleration

out of the vacuum chamber to a pair of near-infrared spectrometers; CCD cameras recorded the spectra on each shot at two different dispersions.

Fig. 5 shows electron energy spectra obtained from shots with helium gas. These shots have been selected to show the trend observed as the density of the gas jet was varied. Electrons were accelerated to relativistic energies at all densities. At low densities $\left(n_{e}<6 \times 10^{18} \mathrm{~cm}^{-3}\right)$ the electron energy spectrum could be characterized by an effective temperature, that is the number of electrons $N$, with energy $E$ was given by $N(E) \propto \exp \left(-E / T_{e f f}\right)$. As the density was increased the maximum energy observed increased, along with $T_{\text {eff }}$. The spectrum also begins to take on a non-Maxwellian form. At $n_{e}=(7.7 \pm 0.7) \times$ $10^{18} \mathrm{~cm}^{-3}$ the acceleration is significantly enhanced, and the energy observed was up above $300 \mathrm{MeV}$. The maximum is, in this case, limited by the noise level on the image plate. As the density was increased above $10^{19} \mathrm{~cm}^{-3}$, the acceleration was observed to be less effective and the spectrum regains its effective temperature form.

Fig. 6 shows explicitly how the acceleration varied with electron density for helium experiments. The effective temperature varies strongly with density exhibiting an apparent peak around $1 \times 10^{19} \mathrm{~cm}^{-3}$. The data taken with deuterium gas produced similar spectra, and show the same variation with density, although the optimum density is shifted slightly to $(1.40 \pm 0.07) \times 10^{19} \mathrm{~cm}^{-3}$. 
Fig. 6 also shows the relationship between the amount of forward Raman scattering (FRS) observed and the electron density. It is clear that the production of high-energy electrons and FRS are uncorrelated. This is in marked difference to previous experiments [4], and indicates that the acceleration mechanism cannot be self-modulated laser wakefield acceleration (SM-LWFA) as in the experiments described in Sec. 2.2.

A study by Gahn et al. [27] with $200 \mathrm{fs,} 0.25 \mathrm{~J}$ pulses showed an enhanced acceleration at $2 \times 10^{20} \mathrm{~cm}^{-3}$. In this experiment there was strong evidence that electron acceleration is strongly correlated with channel formation and magnetic field generation. Simulations indicate that when the betatron motion of the electron in the self-generated magnetic field is resonant with the relativistic motion of the particle in the laser field electron can pick up energy directly from the laser pulse ("direct laser acceleration" (DLA)). This is similar to an inverse free-electron laser mechanism and can occur efficiently when the laser power becomes significantly greater than the critical power for self-focusing.

However the divergence of the beam suggested from these previous lower intensity experiments and simulations is large. With the much smaller divergence observed in the Vulcan Petawatt experiments discussed here the mechanism of direct laser acceleration seems to be more complex and may involve stochastic "de-phasing" processes $[9,28]$ and the combined effect of the laser electric field with the electrostatic field due to charge displacement during the intense laser plasma interaction.

A number of 2D-3V Particle in Cell (PIC) code simulations using the code OSIRIS [29] were subsequently performed showing that a form of direct acceleration by the laser is the dominant acceleration mechanism. The PIC code simulations show the bunching of the accelerated electrons at twice the laser frequency and also that the electrons are accelerated within the laser pulse, the maximum energy coinciding with the maximum laser intensity.

The PIC code simulations have also indicated a possible cause of the observed density-acceleration dependence. Simulations were performed at various densities simulating realistic laser pulses incident on a fully ionized plasma having a linear density ramp from vacuum up to bulk plasma density over $\sim 600 \mu \mathrm{m}$ (this is consistent with the type of nozzle used in these experiments). Each simulation was run for passage throughout the gas jet target. In high density runs $\left(1.4 \times 10^{20} \mathrm{~cm}^{-3}\right)$, the laser undergoes strong self-focusing and filamentation. The filamented laser then undergoes a hosing type instability. This filamentation and hosing does not occur in the low-density case.

If this hosing instability occurred at high densities in the experiment it may be responsible for reducing the effective intensity during the interaction as well as perhaps moving the electron beam off-axis, such that the highest energy electrons did not enter the electron spectrometer (in simulations the electron beam closely follows the laser pulse).

\subsection{Mono-energetic beam production using ultra-short pulses}

Recently electron acceleration experiments using the high power ultra-short pulse Titanium:Sapphire laser system at the Central Laser Facility of the Rutherford Appleton Laboratory (Astra) have been performed [7]. The laser pulses $\left(\lambda=800 \mathrm{~nm}, E=450 \mathrm{~mJ}, \tau_{L}=40 \mathrm{fs}\right)$ were focused with an $f / 16.7$ off axis parabolic mirror onto the edge of a $2 \mathrm{~mm}$ long supersonic jet of helium gas to produce intensities on the order of $1.3 \times 10^{18} \mathrm{Wcm}^{-2}$. The plasma density was varied within the range $n_{e}=3 \times 10^{18} \mathrm{~cm}^{-3}-5 \times 10^{19} \mathrm{~cm}^{-3}$. In this density range the wavelength of relativistic plasma waves produced (i.e., $\lambda_{p}=2 \pi c / \omega_{p e}$ ) is can be less than the laser pulse length $\left(c \tau_{L}\right.$, where $\tau_{L}$ is the laser pulse duration). For laser pulses, which are less than the plasma wavelength, relativistic plasma waves can be generated "resonantly" in the wake of the pulse - while in the regime, in which the laser pulse length is longer than the plasma wavelength, high intensity interactions are required to drive an instability, in which the plasma waves are produced via self-modulation of the laser pulse envelope (i.e., the "wavepacket") at the plasma frequency. In the work described the plasma waves are driven until wavebreaking occurs. Electrons, which reach relativistic energies $\left(v>v_{\phi}\right)$ from their motion in the plasma wave, can out-run other plasma electrons and remain in phase with the plasma wave and gain energy. The "cold" wavebreaking electric-field amplitude for electron plasma waves is given by, $E_{w b}=\sqrt{2\left(\gamma_{p}-1\right)} m_{e} c \omega_{p e} / e$, where $\gamma_{p}$ is the Lorentz factor associated with $v_{\phi}$. Note that in a regime between SM-LWFA and LWFA lies the F-LWFA (forced laser wakefield accelerator), where a short pulse is used, which is only slightly longer than the resonant pulse length [5]. This pulse is consequently focused and compressed by the wave to produce large amplitude plasma waves, which can break.

Note that in the experiment described here the electron energy spectrum was measured using an on-axis magnetic spectrometer similar to that in the Petawatt experiments. The electrons were also simultaneously measured with the high-resolution image plate detectors as well as using a much lower resolution array of diodes. The spectrometer magnet, image plates and diodes were set up to measure the spectrum over a wide energy range in a single shot.

Other diagnostics used in this case included the simultaneous measurement of the transmitted laser spectrum and transverse optical probing of the interaction with a frequency doubled laser probe beam. This was used to produce images of the plasma via shadowgraphy, and was independently timed so it could also be used to measure prepulse effects and plasma channel formation.

Electron acceleration was observed over a range of electron densities. With the plasma density below $\approx$ $1 \times 10^{19} \mathrm{~cm}^{-3}$ no energetic electrons were observed (this corresponds to $c \tau_{L} \leq \lambda_{p}$ ). In this regime, the growth 
rate of the self-modulation instability is too low for a plasma wave to reach wavebreaking amplitudes with the available laser energy. As the density was increased above $1 \times 10^{19} \mathrm{~cm}^{-3}$, very high-energy electrons were produced with the most energetic electrons reaching up to $100 \mathrm{MeV}$. The output beam divergence was also measured and was found to be less than 5 degrees. However the most interesting aspect of these spectra is that, in this regime, the electron energies were exceptionally non-Maxwellian and, indeed, generally consisted of one or more narrow spiky features - each of which could have an energy bandwidth of less than $20 \%$. This is in contrast to the energy spectra of previous laser acceleration experiments, in which $100 \%$ energy spreads are observed. As the density was increased in our experiments, the peak energy of the observed electrons was observed to decrease and the spectra begin to assume a broad shape characteristic of previous experiments in the SM-LWFA regime.

The difference observed in these spectra is evidently due to the timing of the injection of electrons into the relativistic plasma wave. For peaked spectra to be produced, the electrons must be injected into the plasma wave with small spatial and temporal spread. Ideally the electrons have an initial energy much less than the energy gained from the plasma wave, though this can be overcome somewhat by phase rotation of the electron beam, (turning a small spatial but large energy spread, into a small energy spread, but with concomitant increase in spatial dimension). It is apparent that in these experiments where the focal spot dimensions are greater than $\lambda_{p}$, the plasma wave is ideally shaped for such controlled injection.

However at high densities, the interaction length observed in the experiments can be much longer than the dephasing length - the length over which an electron outruns the plasma wave and begins to be deaccelerated by the wave $-L_{d}=2 \pi c \omega_{L}^{2} / \omega_{p e}^{3}$, where $\omega_{L}$ is the laser frequency. This means that any initially localised bunches will rapidly be randomised by over-rotation in phase space, leading to the large energy spreads observed experimentally. Furthermore, at high densities the plasma wave evolution is so rapid, that the injection is unlikely to be particularly localised in any case.

For a density of $2 \times 10^{19} \mathrm{~cm}^{-3}$, the dephasing length is on the order of the observed interaction lengths, which is about $1 \mathrm{~mm}$. Indeed above this density, broad spectra are seen, whereas at around this density and below, strongly modulated spectra are seen. The multiple spikes observed at low densities are likely to be due to multiple injection into the first plasma wave cycle, or injection into trailing wavebuckets of the wakefield.

In a further experimental run the energy of the laser pulse was increased to about $500 \mathrm{~mJ}$. These experiments showed that for the densities used to obtain "bumpy" spectra in the lower energy situations that very "monoenergetic" spectra could be observed. This is shown in Fig. 7, in which 2 electron spectra are shown - from the same series of shots. The spectra are reasonably re-

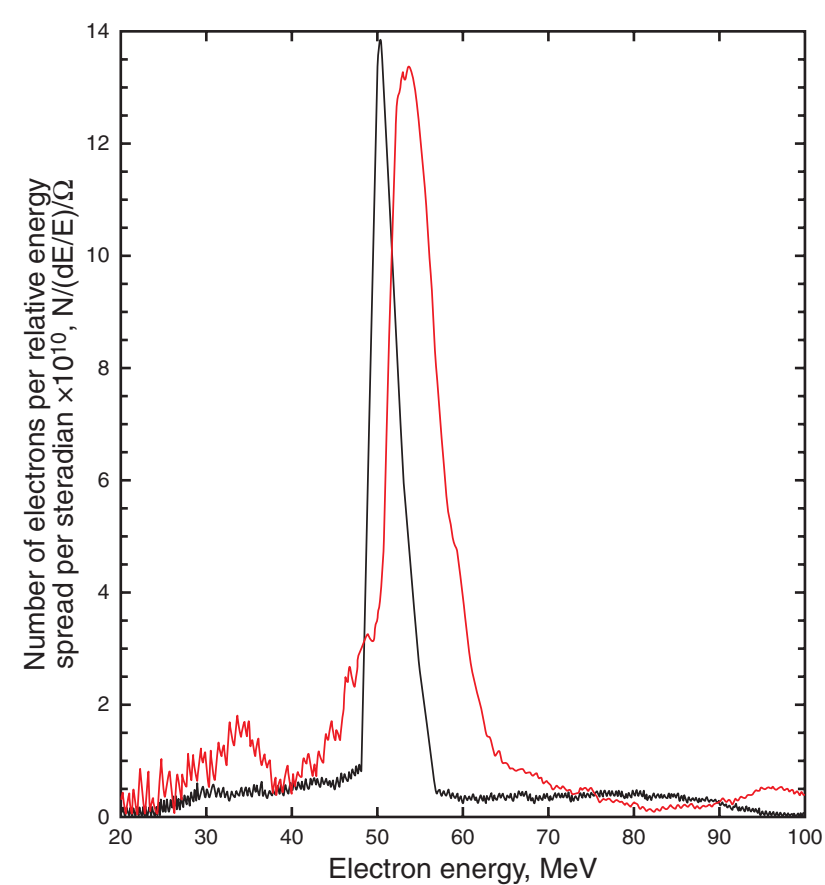

Figure 7 (online color at www.lphys.org) Two measured electron spectrum with $E=500 \mathrm{~mJ}$ laser at a density of $2 \times 10^{19} \mathrm{~cm}^{-3}$. Shots are taken from the same shot series

producible and the narrowest spectrum shows a beam at $52 \mathrm{MeV}$ with an energy spread $(\Delta E / E)$ of less than $5 \%$.

These observations are reproduced in $2 \mathrm{D}$ particle-incell simulations of the interaction were performed using the code OSIRIS. A high power short pulse laser pulse focused to a spot size initially greater than the relativistic plasma waves wavelength $\lambda_{p}$, will be amplified by the combined action of temporal compression [30], photon acceleration [14] and most importantly self-focusing [10]. This can gradually cause the wakefield created by the laser pulse to increase in amplitude to the point that self-injection can occur. Self-injection can prevent further growth of the plasma wave and means that the plasma wave is not destroyed, a process sometimes called adiabatic wavebreaking [31,32]. The injected bunch is thus localised both spatially and temporally, which as mentioned before, is ideal for them to be uniformly accelerated, resulting in monoenergetic bunches.

This process of gradual pulse shaping, resulting in a instantaneous injection and violent acceleration of a bunch of particles is supported by recent observations of the synchrotron radiation emitted by the localised bunch of electrons injected into the wakefield [33]. As the laser pulse travels through the plasma, it initially scatters strongly in the transverse direction by Raman processes. This pulse erosion helps shaping of the pulse shape, in particular increasing pulse compression up to the point that the pulse fits well within the caviton formed by its ponderomo- 


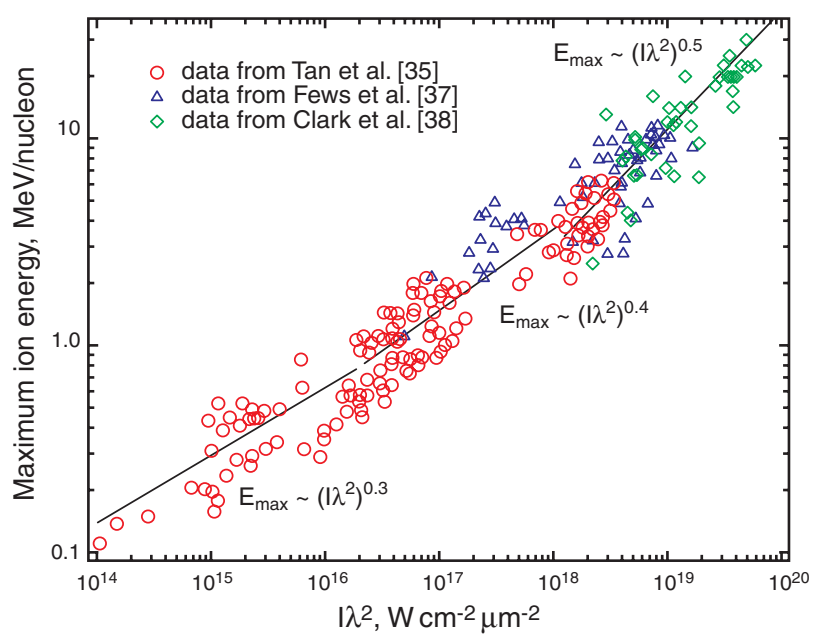

Figure 8 (online color at www.lphys.org) Maximum ion energy against irradiance on target. In addition, heavy ions were measured using a Thomson parabola and CR-39 detector

tive force (the so-called "bubble"). This actually increases pulse amplification, and self-injection occurs, with the simultaneous observation of a short but broadband pulse of radiation that is, unlike the Raman signal, polarisation independent. The radiation is due to the coherent synchrotron radiation of micro-bunched structure within the electron bunch generated by self-injection, which is strongest as the bunch is initially accelerated from rest when it is predominantly in the transverse direction. Hence transverse images of this broadband radiation can diagnose the exact position, at which injection occurs. It is found experimentally that injection occurs after a shorter propagation distance as the gas-jet density is increased, which is consistent with simulation (and expectation) that pulse evolution is quicker at higher density.

It is clear then that the bunches of electrons are produced due to wavebreaking. These electrons are then accelerated through the entire length of the plasma - which is shorter than the dephasing distance. Consequently, the bunch of electrons can remain relatively mono-energetic after leaving the plasma. The requirements for this regime are that the plasma density has to be high enough so that wavebreaking is easily achieved for an interaction at a given density - but low enough so that the electron bunches produced are not de-phased before they leave the plasma.

To summarise, it has been demonstrated that ultra short pulse lasers can be used to produce relativistic bunches of electrons with energies up to $100 \mathrm{MeV}$. Accelerated electrons are not observed below a minimum density - but at densities slightly higher than this "mono-energetic" electron beams can be clearly observed in the spectrum. As the plasma density is increased still further such structures are randomised by the dephasing of the accelerated electrons with the plasma waves and the energy spread of $100 \%$ is observed.

The observation that laser produced plasmas alone can produce mono-energetic electron beams suggests that such sources hold great promise for future development of table top particle accelerators and that a wide range of applications may soon become possible.

\section{Ion acceleration using short laser pulses}

In this section, recent measurements of energetic ion emission from intense laser interactions with plasmas will be discussed (from both solid and gaseous density interactions).

\subsection{Observations of ions from the "front" of laser solid interactions}

Some of the first plasma physics experiments to be performed using high-powered lasers were energetic ion measurements [34-36]. Such experiments have always been important for the elucidation of the highly transient, nonlinear phenomena, which occur when an intense laser pulse interacts with a plasma. The beginning of research into inertial confinement fusion (ICF), together with development of multi-kilojoule lasers initially brought about considerable interest in these experiments. Indeed studies of ion production from planar and spherically irradiated targets are important for diagnosing the coupling of laser radiation into hot dense plasmas as well as the efficiency of plasma compression of by lasers for ICF.

Results from experiments [35] in the early 1980's using long wavelength $\mathrm{CO}_{2}$ lasers indicated that ions with energies greater than $1 \mathrm{MeV}$ could be produced. However, such work was undertaken using long pulse lasers (durations of $\sim 1 \mathrm{~ns}$ ) unlike the sub-picosecond duration laser pulses available today. Both theoretical and computational studies at the time [34] were able to model the plasma expansion from the target surface and the subsequent acceleration of ions in the plasma. The self-similar solution for the isothermal expansion of the laser-produced plasma indicated the presence of a space-charge electric field, which is able to accelerate the ions as the electrons expand into vacuum (sheath acceleration) [36]. This mechanism produces a maximum ion energy - i.e., a high-energy cut-off - which was similar to the experimentally observed ion energy distribution. Indeed, the maximum ion energy has also been shown experimentally to be related to the hot electron temperature agreeing with the sheath acceleration mechanism.

Accumulated data from a number of published experiments over the past thirty years is shown in Fig. 8. Of interest is the maximum proton/ion energy for interactions above $10^{18} \mathrm{~W} / \mathrm{cm}^{2} \mu \mathrm{m}^{2}$, which is found to scale as $\left(I \lambda^{2}\right)^{0.5}$ i.e., when the oscillatory velocity of the electrons 


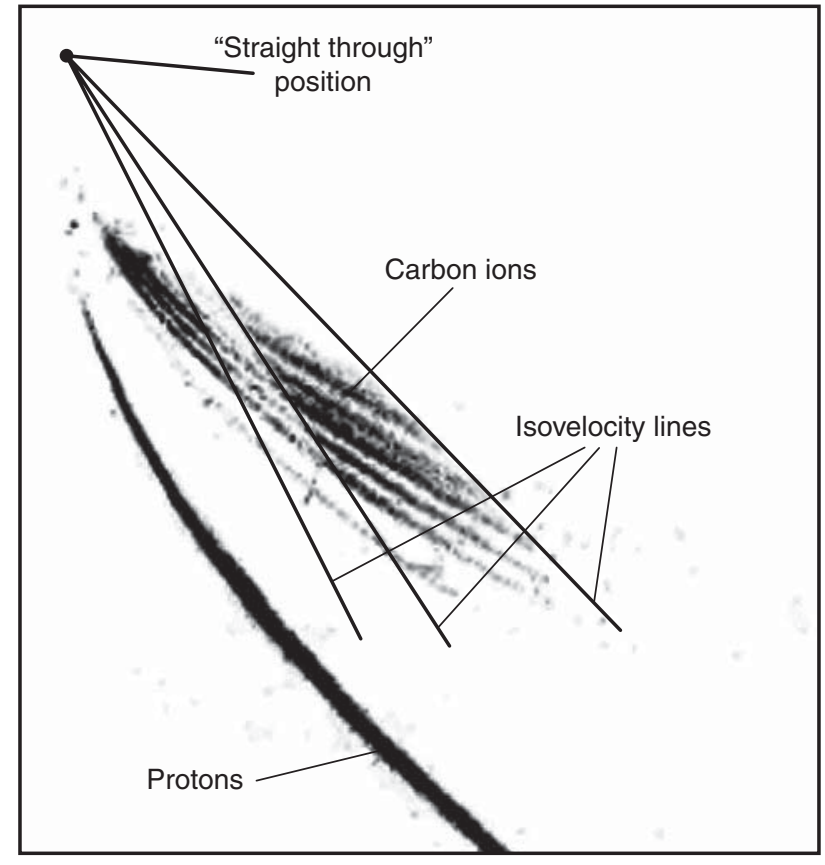

Figure 9 Raw data from CR39/Thomson parabola ion spectrometer

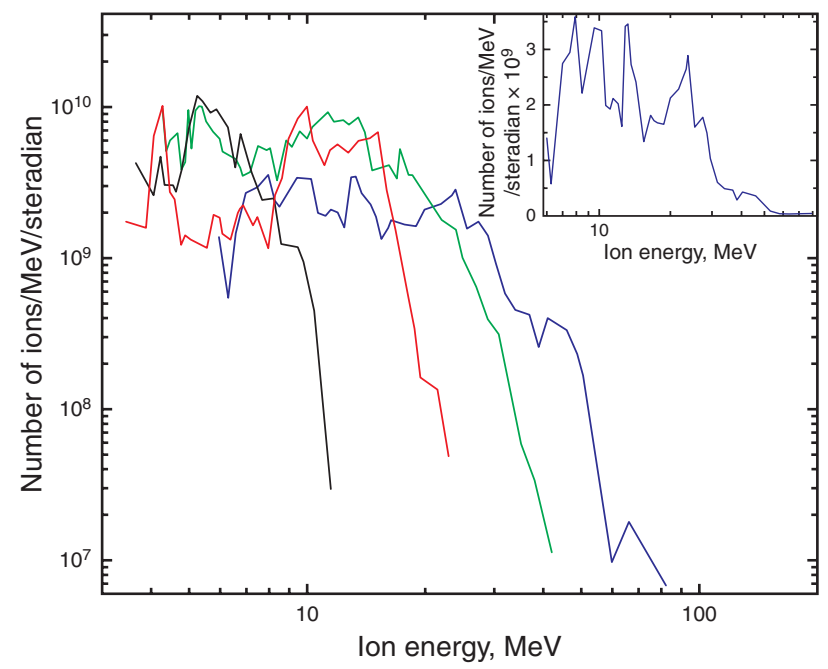

Figure 10 (online color at www.lphys.org) Typical carbon spectrum from front surface interaction

in the laser field becomes relativistic - which is similar to the observed trend for the hot electron temperature in this regime. This suggests that the laser energy is mainly coupled to the plasma via ponderomotive $\mathbf{J} \times \mathbf{B}$ acceleration of electrons at these intensities.

In the short pulse regime ( $\sim 1 \mathrm{ps})$ the first ion measurements made at the front of the target were found to be in general agreement with the earlier results from long pulse lasers in terms of the scaling of the maximum ion energy as a function of laser intensity [37]. These ion spectra exhibited a sharp cut-off at energies up to $12 \mathrm{MeV}$.

Subsequent observations of the proton emission at the front of the target at high intensities indicated that there is significant structure in the proton spectrum as well as an angular emission profile, which is non-uniform and which varies with energy. Two dominant components to the spatial distribution have been observed [38]. At lower energy, a ring of ablated plasma is observed, which contains low ( $<4 \mathrm{MeV} /$ neucleon) energy ions/protons. This ring-like emission is formed at the target surface in the ablating plasma and is likely to be caused by self-generated magnetic fields in the plasma and the subsequent influence of this field on electron transport along the target surface. At higher energies, the proton emission has a qualitatively different spatial distribution and is much more diffuse. It is likely that the higher energy protons are accelerated by a combination of a "Coulomb explosion" generated by the laser during the interaction as well as the accelerating "sheath" fields produced by the hot electron population, which escapes the plasma.

The protons from such interactions originate from hydrocarbon/water vapor contaminants on the surface of the target (which exist within almost every vacuum system) as well as some from within the target material itself. In Fig. 9 a scanned image of a piece of CR39, a nuclear track detector, is shown which has been used as the detection medium for a Thomson parabola ion spectrometer. This was produced from an interaction of the Vulcan 50 TW $\mathrm{Nd}$ :Glass laser with a $2 \mathrm{~mm}$ thick lead target at an intensity at about $2 \times 10^{19} \mathrm{Wcm}^{-2}$ at RAL. The target was oriented at $45^{\circ}$ to the incident direction of the laser. The ion spectrometer was positioned in front of the target and offset from the target normal by $15^{\circ}$. This diagnostic enabled the heavier ions as well as the protons emitted from the target surface to be measured. The proton spectrum derived from this data (not shown) for this case exhibits an exponential fall-off in the number of protons up to $4 \mathrm{MeV}$ followed by a flattening of the spectrum to the diagnostic limit of $11 \mathrm{MeV}$. From this spectrum - given the angular dispersion of the protons, it can be estimated that there are $\sim 10^{12}$ protons having an energy greater than $500 \mathrm{keV}$. The total energy in the proton "beam" emitted from the front surface of the target is about $5 \%$ of the total laser energy incident onto the target. By fitting an exponential $(\exp (-E / T))$ to the spectrum in the energy region less than $4 \mathrm{MeV}$, an ion temperature of $700 \mathrm{keV}$ is obtained.

An interesting observation is that the proton spectrum often exhibits modulations, which are up to twice the background "continuum" level. These peaks in the spectrum are reproducible but vary in position from shot to shot and the energy "width" of the peaks is determined by the resolution of the ion spectrometers used.

The spectrum of the carbon ions can also be obtained from the piece of CR39 as shown in Fig. 10. Ions with charge states up to $\mathrm{C}^{6+}$ are present and it can be seen that carbon ions having an energy up to $100 \mathrm{MeV}$ are observed 
and that the maximum energy occurs for those ions with the highest ionization state. The carbon ions also have a modulated structure, similar to the protons but at a lower energy per nucleon. This is more apparent in the inset in Fig. 10 where the $\mathrm{C}^{6+}$ spectrum is inset and plotted on a linear scale.

This can also be seen in the raw data shown in Fig. 9. Along the carbon parabolas (ions are separated into different "parabolas" according to their charge/mass ratio in this type of ion spectrometer), distinct "band" like structures can be observed, which indicate changes in the density of the number of pits along the parabolas. The bands occur between imaginary lines, which pass through the "straight through" position. These lines indicate loci of constant velocity, which suggest that the carbon ions are accelerated in "velocity bunches" and acquire similar velocities despite having different charge states. It should be noted that the highest energy ions of each species lie along the line, which indicates a constant energy per charge state - as if the highest energy ions are accelerated by falling through the same potential (i.e., before the number of accelerated ions acts to reduce the accelerating fields in the sheath).

Lead spectra obtained during the same shot as the carbon and proton spectra were also obtained. From this data, $\mathrm{Pd}$-like $\mathrm{Pb}^{36+}$ ions up to $220 \pm 30 \mathrm{MeV}$ and $\mathrm{Kr}$-like $\mathrm{Pb}^{46+}$ ions up to $430 \pm 40 \mathrm{MeV}$ were measured with the Thomson parabola diagnostic. The quoted charge state represents an upper limit to the charge state, which could be measured on the CR39. Ions of lower charge were also present, but individual parabolas could not be resolved on the detector. As in the case of the carbon ions, the ion species having the highest energy is the one with the highest charge state.

Targets can also be heated radiatively to more than $400^{\circ} \mathrm{C}$ using a heating element positioned near the target. The temperature was monitored using a thermocouple and was maintained at a constant level prior to the shot. The purpose of heating the target was to investigate the source of the protons in metallic targets (by removing the surface layers of contaminants through evaporation) and to observe the influence of removing the hydrogen on the acceleration of the heavy ions. The number of accelerated protons was substantially reduced by up to two orders of magnitude when compared to interactions with unheated gold or aluminum targets at a similar intensity of $2 \times 10^{19} \mathrm{~W} / \mathrm{cm}^{2}$. The maximum carbon ion energy was increased by about $20 \mathrm{MeV}$ and the population of these ions was pushed towards the high-energy end of the spectrum while the proton number and maximum energy were radically reduced. Indeed the reduction of the number of accelerated protons and the enhancement in the number and energy of heavier species has been confirmed by the use of nuclear activation diagnostics [39].

It is clear that very energetic ions can be generated at the front surface of the interaction of a high intensity laser with a solid target. It is likely that fast proton acceleration occurs near the critical surface by space charge acceleration from the hot electrons generated in this region and it is possible that a complex hot electron spectrum resulting from the laser plasma interaction would manifest itself as modulations in the proton and heavier ion spectrum. However the modulation features in the proton and heavier ion spectra, which vary in position from shot to shot and the complex emission pattern of these ions make these ions difficult to use for many applications.

\subsection{Ions and protons from laser interactions with thin foil targets}

The first measurements of ion acceleration in the "forward direction" i.e., in the direction of the laser propagation, were inferred from neutron measurements of beamfusion neutrons from the interaction of intense laser pulses with deuterated targets [40,41]. It was determined that deuterium ions with energies of at least several hundred $\mathrm{keV}$ could be accelerated into the target. These conclusions were obtained from measurements of the angular distribution of the neutrons from beam-fusion D-D interactions in the target. Modelling of these interactions has shown that the ponderomotive pressure of the laser can expel ions into the target to energies of a few $\mathrm{MeV}$ at these intensities. This process bores a hole into the overdense plasma and indeed can generate an electrostatic shock, imparting even more energy to the ions [42].

A number of more recent measurements have directly recorded the production of proton and ion beams at the rear of thin solid targets irradiated by a high intensity laser beam. In Vulcan experiments at the Rutherford Appleton Laboratory protons with energies up to about $40 \mathrm{MeV}$ were measured at the rear of the target. The laser wavelength was $1.054 \mu \mathrm{m}$, the pulse length was $0.9-1.2 \mathrm{ps}$ and the incident energy on target was up to $100 \mathrm{~J}$. The laser beam was focused onto the target surface using a $f=225 \mathrm{~mm}$ on-axis parabolic mirror and was $p$-polarized incident at an angle of $45^{\circ}$. The peak intensity was more than $10^{19} \mathrm{~W} / \mathrm{cm}^{2}$.

The targets used were generally aluminium, ranging in thickness from $10 \mu \mathrm{m}$ to $1 \mathrm{~mm}$, cut into $5 \mathrm{~mm}$ by $5 \mathrm{~mm}$ squares. Behind each target, a CR39/RCF diagnostic stack was placed at a distance such that the entire beam of the particle emission could be captured by the diagnostic. This stack typically consisted of a piece of $110 \mu \mathrm{m}$ thick RCF followed by 3 or more pieces of $0.75 \mathrm{~mm}$ thick CR39. The unique feature of this passive CR39/RCF stack diagnostic is that it is capable of simultaneously measuring the spatial distribution and the energy of the protons (and electrons) and was first used during these experiments [43]. The diagnostic was placed about $25 \mathrm{~mm}$ behind the target as shown in Fig. 11. Quantitative measurements of the spatially integrated proton spectrum can be obtained using a similarly designed copper activation stack diagnostic [44].

Ions deposit energy in the CR39 as they pass through and damage the plastic. Most of the energy is deposited just as the proton stops at the Bragg peak. CR39 is subsequently developed in a $\mathrm{NaOH}$ solution that etches a pit 


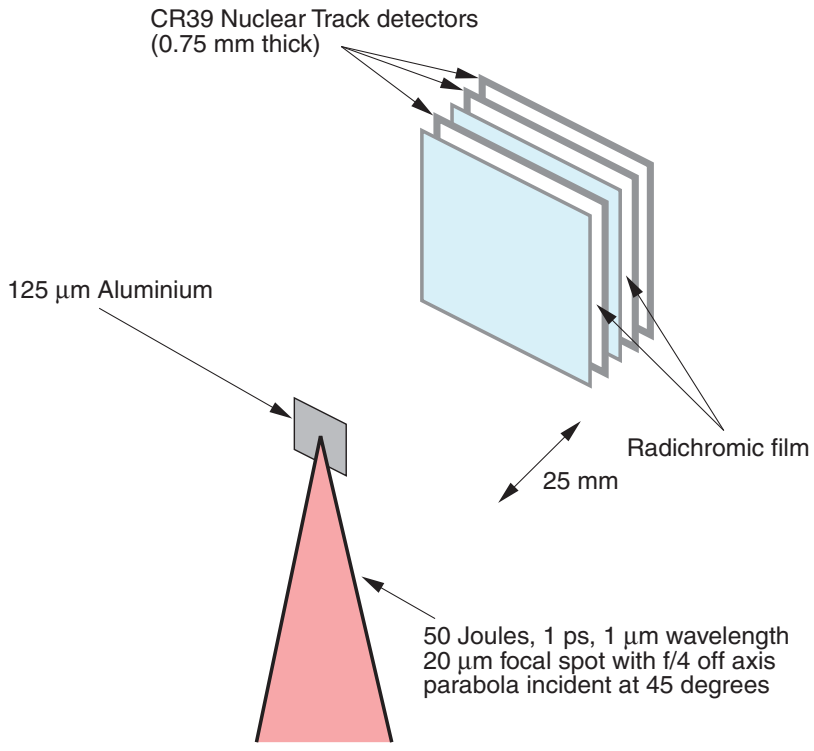

Figure 11 (online color at www.lphys.org) Experimental setup for measurement of ion emission from rear surface of thin foil targets

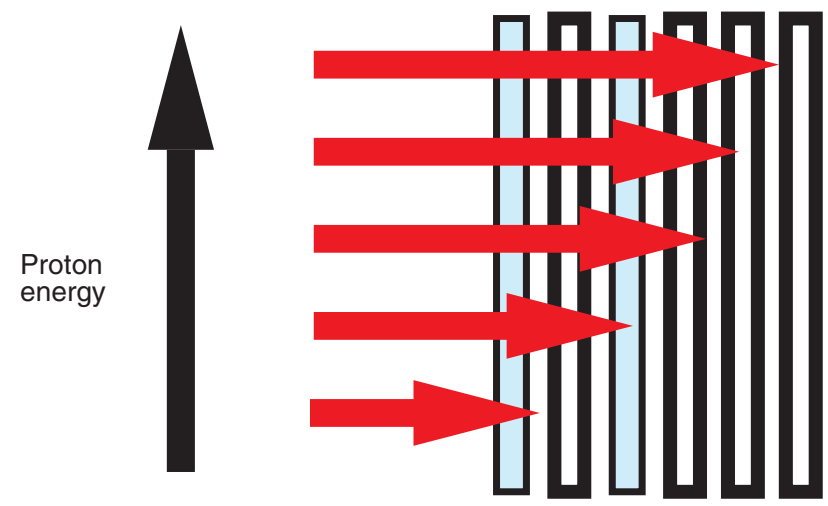

Figure 12 (online color at www.lphys.org) Schematic of use of "sandwich" detectors for proton spectroscopy

at the surface of the plastic if a proton was stopped there. The particular advantage of this diagnostic is that knowledge of the range of protons in CR39 and radiochromic film enables the energy of protons, which have produced etched pits to be determined as the protons pass through the stack (see Fig. 12).

Protons with comparable energies to those observed from the front surface are observed from the rear surface too. A particularly interesting aspect of these first measurements was the observation that for some targets the protons were emitted in quasi-mono-energetic "ring" structures such that a narrow range of energies were emitted primarily at a particular angle and such that the ring radius decreased with increasing proton energy. The production of this ring structure has been confirmed through the use of other diagnostic techniques such as nuclear activation [45].

The maximum divergence of this ring pattern was up to $60^{\circ}$. The ring pattern may be caused by deflection of the protons by magnetic fields within the target interior if one considers that the origin of these protons could be the front surface. Magnetic fields inside the target can be generated by the current of hot electrons that also propagate into the target ahead of the protons. The spatial distribution of the protons as measured by the CR39/RCF diagnostic is shown in Fig. 13. This data was taken from a shot with a laser intensity of $2 \times 10^{19} \mathrm{~W} / \mathrm{cm}^{2}$ incident on a $125 \mu \mathrm{m}$ thick aluminium target. Fig. 13a shows a scanned image of the front piece of radiochromic film. The signal on the film is contained within a well-defined radius from the central burn mark in the centre of the film. The angle subtended by the periphery of this signal covers a cone half angle of $30^{\circ}$. The center of the film is coincident with the rear target normal. Towards the center of the film, as the radius decreases, there is an abrupt change in optical density on the film at a cone half angle of $10^{\circ}$, while at the center, the film has been burned.the film has been damaged.

Fig. 13 also shows the scanned images of the surfaces of the CR39 facing the target. The CR39 is only sensitive to protons and ions, however ions of species other than protons would be unlikely to pass though the first layer of $\mathrm{RCF}$. The signal observed in these images is consequently predominantly due to protons. Observation of the size of the pits indicated that they were $\sim 10 \mu \mathrm{m}$, which for these etching conditions would also confirm that the tracks were made by protons. Fig. 13 indicates that most of the proton signal is situated on the ring. From the stopping range of the protons in the diagnostic, it can be estimated that these protons have an energy of $3 \pm 1 \mathrm{MeV}$. The signal on the CR39, which forms the ring is saturated but an estimate of the number of protons can be derived by comparison of the CR39 signal with the outermost region of the RCF signal in Fig. 13a. Clearly, there is a strong correlation between these two regions so it can be estimated that there are $\sim 10^{12}$ protons with energies in excess of $2 \mathrm{MeV}$. The contrast between the signal (protons $/ \mathrm{cm}^{2}$ ) contained in the ring and the signal inside the ring, which is not saturated is about $10^{4}: 1$. Fig. $13 \mathrm{c}$ to Fig. 13e show the surfaces of the layers of CR39 further into the stack. As the energy of the protons increases, the radius of the ring pattern decreases, until at $\sim 18 \mathrm{MeV}$, the proton signal is reduced to a "dot". In effect, as a function of angle from the rear target normal, a mono-energetic beam of protons has been measured, which has rotational symmetry around the rear target normal. Clearly, this is an unusual observation, where it might have been intuitively expected that a broad spectrum of ions would be observed at each emission angle. If this was the case, then the signal on the CR39 would manifest itself as a disk of signal rather than a ring.

It is important to note that the actual signal levels in the interior of the rings shown here are not overexposed and indeed individual proton tracks can be observed and 


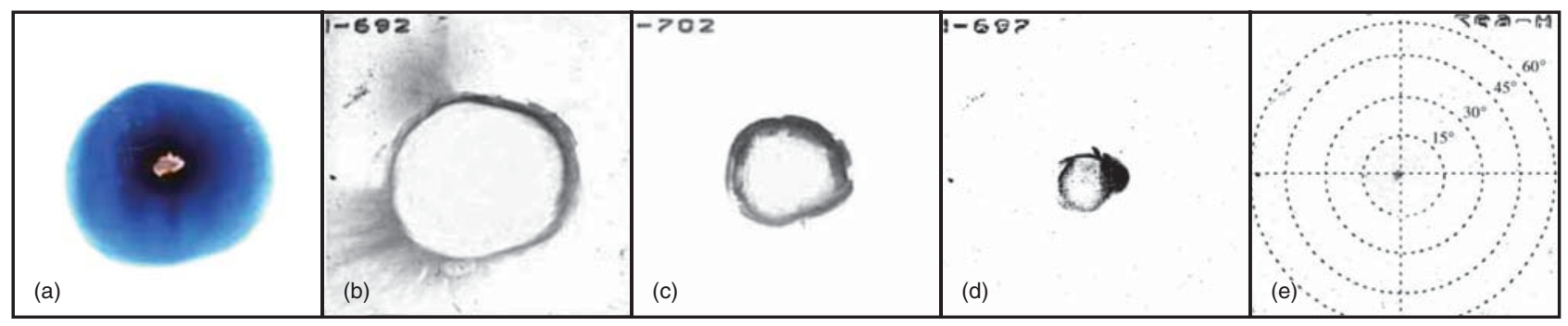

Figure 13 (online color at www.lphys.org) CR-39 track detectors showing a ring-like structure and that the proton beam is emitted in a mono-energetic pattern that is energy dependent

counted within the structure - although the signal level in the "ring" itself is saturated. The ring structure is, in fact, not observable when the proton signal was highest - i.e., for thin targets $(<50 \mu \mathrm{m})$ when in fact the emission pattern was observed to be uniform - but rather only occurred when the target was thicker $(50-200 \mu \mathrm{m})$ such that the proton signal levels were reduced. The fact that the ring structure can be reproducibly observed only for "thicker" targets is important evidence that this structure may be due to relatively low fields within the target.

The emission pattern of protons emitted from very thin targets is more uniform and less divergent than that from the thicker target discussed above. The number of protons produced also increases significantly as the target is made thinner until the energetic proton emission from the rear exceeds that observable at the "front" of the target. Indeed when thin $(<50 \mu \mathrm{m}) \mathrm{Al}$ foil targets are irradiated with $5 \times 10^{19} \mathrm{Wcm}^{-2}$ on target (using the Vulcan 100 TW facility) a uniform poly-energetic proton distribution is observed with no ring structure present.

There have been a number of experiments [43-48], which have subsequently investigated the acceleration of protons from the rear surface of very thin foil targets - and such beams seem to be emitted with unusual beam qualities. Several experiments have shown that acceleration of these protons from the rear surface of the thin foil is the dominant mechanism in such experimental situations. This mechanism is the well known sheath acceleration, which causes most of the observed acceleration of ions at the ablated plasma at the front. In this case the process occurs within a plasma created at the rear surface by the hot electrons, which propagate through the target. Such acceleration can be well simulated using collisionless particle in cell codes and these codes suggest that the direction the proton emission can be affected by the shape of the rear surface and that it may be possible to "focus" this proton beam in order to deposit the energy into a small region of plasma for inertial confinement fusion applications (i.e., fast ignition and isochoric heating of plasma).

Indeed for targets composed of conducting materials the proton beam quality is much superior to that from insulating materials - which is indicative of improved hot electron flow through the material. This indicates that sheath acceleration from the rear surface may be dominant for those conditions, in agreement with the observations of Snavely et al. [46] who have observed the highest reported accelerated proton energies $(58 \mathrm{MeV})$.

Depending on the laser intensity and the target thickness, simulations suggest that front surface (due to collisionless shock acceleration) and rear surface (due to sheath acceleration) mechanisms can become dominant in different experimental configurations. It is interesting to note that acceleration in the shock becomes dominant when the protons accelerated in this way reach the sheath acceleration region at the rear side of the target with a velocity greater than the ions already accelerated in the sheath region. Particle-in-cell modelling by Silva et al. [42], suggests that a signature for this effect is the formation of a plateau region between $15-30 \mathrm{MeV}$, which can be clearly observed in data from various experiments. It is also noted that the initial plasma temperature and target resistivity also plays a significant role in determining, which process dominates.

One of the obstacles for applications of these beams is that the beams are generated with a broad energy spread consequently methods to produce mono-energetic sources could be important for the development of these applications. Clearly the observation of energy resolved angular distribution in Fig. 13 indicates that it is possible to generate mono- energetic proton beams rather easily with the insertion of a suitably designed filter (aperture). It has also been demonstrated that careful control over the target conditions can also give rise quasi-monoenergetic beams of both protons and ions [47] from the back of thin foil targets.

These protons could then be used as an injector for subsequent acceleration in a conventional accelerator. Indeed, heavier ions could also be used for this purpose in the future. It is interesting to note that Cowan et al. [48] have shown that the emittance of protons from a laser-produced plasma source can be less than $0.004 \pi \mathrm{mm}$ mrad, which is two orders of magnitude better than conventional injectors for accelerators. Heating the target also removes the proton layer from the rear of the target and enables the acceleration of heavier ion species [49]. 


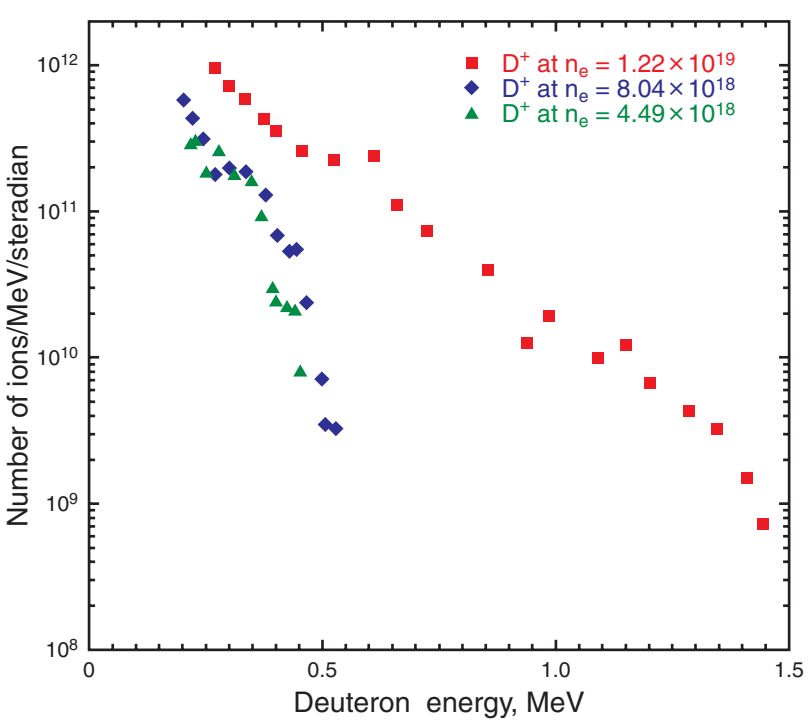

Figure 14 (online color at www.lphys.org) Measured spectrum of deuterium ions resulting from intense laser interaction with deuterium gas jet targets

\subsection{Ions from laser interactions with underdense plasmas}

Another potentially important source of energetic ions are those resulting from the interaction of intense laser beams with underdense plasmas. However in this case the energetic ions are typically produced primarily perpendicular to the direction of propagation - although recently we have made measurements of ions accelerated in the direction of laser propagation using the Vulcan Petawatt laser [50].

The acceleration mechanism in the radial direction is also fundamentally different. The large ponderomotive force of the laser pulse acts to displace electrons in the plasma and set-up an electric field because of charge separation. Since the three dimensional shape of the focused laser pulse is typically longer than it is wide (i.e., "cigar" shaped) this implies that the electric field experienced by the ions in the focal volume during the passage of the pulse is primarily radial. Consequently, the pulse length of the incident laser will determine the duration of the acceleration. In experiments the radial acceleration of these ions is the principal observation [51]. The acceleration of ions using this technique is termed either as "Coulomb explosion" because of the space charge fields resulting from the charge displacement caused by the ponderomotive force of the laser pulse during the interaction or alternatively "ponderomotive shock acceleration".

Study of the ion dynamics is important as it can supply valuable information of the fundamental physics of the interaction of a high-intensity laser with underdense plasma, such as self-focusing and channelling due to relativistic and charge displacement effects. It is also directly related to the observations of anomalously high yields of neutrons resulting from hot channel formation [52]. The maximum ion energy that can be produced by this process is roughly equal to the ponderomotive energy. Recently measurements of energetic ions accelerated during the interaction of a $0.25 \mathrm{PW}$ laser at RAL with a gas-jet plasma at electron densities up to $1.4 \times 10^{20} \mathrm{~cm}^{-3}$. For these investigations, the laser produced pulses with an energy up to $180 \mathrm{~J}$ in a duration of $0.5-0.7 \mathrm{ps}$. The laser pulse was focused onto the edge of a supersonic gas jet $(2 \mathrm{~mm} \mathrm{noz-}$ zle diameter) using an $f=3$ off-axis parabolic mirror to a focal spot size of $10 \mu \mathrm{m}$ in vacuum.

The energy spectrum of the ions at 100 degrees from the laser propagation direction was measured with a Thomson parabola ion spectrometer, positioned $80 \mathrm{~cm}$ from the interaction region. The ions were recorded on a $1 \mathrm{~mm}$ thick piece of CR39 nuclear track detector. The angular distribution of the ions was measured with a stack of several layers of radiochromic film (RCF) strips parallel to the direction of laser propagation placed at a distance of $6 \mathrm{~cm}$ radially from the interaction region. The angular distribution of ions in different energy ranges was determined through the use of aluminium filters of various thicknesses in front of the RCF. The spectra show two main characteristics: i) the maximum ion energy and the number of energetic ions are higher at high density; ii) at high density, a plateau is observed at high ion energy. In the high-density case both $\mathrm{He}^{2+}$ and $\mathrm{He}^{1+}$ ions have been accelerated to high energy. The maximum energy for $\mathrm{He}^{2+}$ being $13.2 \pm 1.0 \mathrm{MeV}$. The total number of $\mathrm{He}^{2+}$ ions with energy greater than $680 \mathrm{keV}$ is $3.8 \times 10^{11}$. The maximum ion energy drops to $2.3 \mathrm{MeV}$ and the total number of $\mathrm{He}^{2+}$ ions with energy greater than $590 \pm 20 \mathrm{keV}$ is reduced to $7.4 \times 10^{10}$. At even lower density, i.e., below $4 \times 10^{18} \mathrm{~cm}^{-3}$, no energetic helium ions (greater than $100 \mathrm{keV} /$ nucleon - the detector threshold) were observed. The $\mathrm{He}^{2+}$ ion spectrum exhibits a plateau in the energy range between $6-10 \mathrm{MeV}$ and has a cut-off at the maximum ion energy recorded on the detector. The plateau structure is a characteristic of the acceleration due to laser driven laminar shock waves [42]. An example of an ion emission spectrum due to an interaction with deuterium is shown in Fig. 14. These ions were measured using a Thomson parabola ion spectrometer positioned at 90 degrees from the direction of laser. The ion emission at low density $\left(1.0 \times 10^{19} \mathrm{~cm}^{-3}\right)$ is predominantly at 90 degrees. For energies greater than $2 \mathrm{MeV}$, there is a narrow lobe with an angular spread of less than 4 degrees (FWHM). At higher plasma density, the angular spread is much greater. Even the high energy component $(E>3.5 \mathrm{MeV})$, which has a less broad angular emission than lower energy ions, has an angular spread of 27 degrees, and is preferentially emitted slightly forward of 90 degrees to the laser propagation direction.

By studying the radial momentum of the ions as a function of radial distance in 2D simulations of these interactions, it is possible to show that these features can be explained by the interaction of collisionless shocks. Large populations of ions are seen to be accelerated to higher en- 
ergies especially during the merging of the multiple shocks in the simulations. This is shown most clearly by an abrupt increase in the radial momentum of the ions at radial displacements well beyond the laser beam radius and thus beyond the influence of its ponderomotive force. This acceleration process contributes to the formation of the plateau out to higher energy in the ion energy spectrum, as has been similarly observed in simulations above critical density. The strong dependence of the ion acceleration on plasma density can be explained by the additional shock acceleration at high density, while such mechanisms are progressively less effective with decreasing density.

\section{Conclusions}

The results described above - represent a watershed for work on plasma electron acceleration. It is now clear that plasmas can produce high quality electron beams on their own - such that table top $\mathrm{GeV}$ accelerators have now become possible. With regard to relativistic electron acceleration for low-density plasmas the experiments on the Vulcan Petawatt facility have also successfully accelerated electrons up a maximum energy above $300 \mathrm{MeV}$ with low divergence. However the electron beams produced in this way have a very large energy spread making them not particularly useful for applications - except as an efficient source for MeV radiography [53].

On the other hand a reduction in the pulse length (and increase in the focal length) has shown that the SM-LWFA moves into a completely different regime, in which relativistic "mono-energetic" electron beams can be produced. This seems to be the most attractive route for further scientific exploration as well as for the development of applications.

There are also many applications proposed for the recently observed proton and ion beams from thin foil targets [54]. These include injectors for subsequent conventional accelerators; isotope production for positron emission tomography [44]; fusion evaporation studies [55], among others. Most of the potential applications require improvements in the laser technology or improvements in the understanding of the physics of ion acceleration in order to make the applications economically viable. Indeed for many applications there is a particular requirement for mono-energetic source and there are several researchers who have proposed methods and target configurations to generate such sources [43,47].

However the most significant application of this phenomenon occurred almost immediately after the discovery of these high quality ion beams - primarily because of the excellent beam quality of the proton beams emitted from the rear surface of these thin foils enables them to used very successfully as high resolution probes of electric fields of secondary laser produced plasmas. There have been a number of interesting observations using this technique including the measurement of solitons [56] in a laser-produced plasma. This application is still under development since in fact the probing images are affected not only by electric fields in the target but also in some circumstances by plasma density variations and magnetic fields.

Clearly, with so many potential applications much further work is necessary to develop these sources. It is vital to understand in detail the dependence of different acceleration mechanisms on both the laser and target conditions.

\section{References}

[1] T. Tajima and J.M. Dawson, Phys. Rev. Lett. 43, 267 (1979).

[2] P.A. Norreys, K.M. Krushelnick, and M. Zepf, Plasma Phys. Control. Fusion 46, B13 (2004).

[3] M. Tatarakis, I. Watts, F.N. Beg, E.L. Clark, A.E. Dangor, A. Gopal, M.G. Haines, P.A. Norreys, U. Wagner, M.-S. Wei, M. Zepf, and K. Krushelnick, Nature 415, 280 (2002).

[4] A. Modena, Z. Najmudin, A.E. Dangor, C.E. Clayton, K.A. Marsh, C. Joshi, V. Malka, C.B. Darrow, C. Danson, D. Neely, and F.N. Walsh, Nature 377, 606 (1995); C.A. Coverdale, C.B. Darrow, C.D. Decker, W.B. Mori, KC. Tzeng, K.A. Marsh, C.E. Clayton, and C. Joshi, Phys. Rev. Lett. 74, 4659 (1995); K. Nakajima, D. Fisher, T. Kawakubo, H. Nakanishi, A. Ogata, Y. Kato, Y. Kitagawa, R. Kodama, K. Mima, H. Shiraga, K. Suzuki, K. Yamakawa, T. Zhang, Y. Sakawa, T. Shoji, Y. Nishida, N. Yugami, M. Downer, and T. Tajima, Phys. Rev. Lett. 74, 4428 (1995); D. Umstadter, S.-Y. Chen, A. Maksimchuk, G. Mourou, and R. Wagner, Science 273, 472 (1996); A. Ting, C.I. Moore, K. Krushelnick, C. Manka, E. Esarey, P. Sprangle, R. Hubbard, H.R. Burris, R. Fischer, and M. Baine, Phys. Plasmas 4, 1889 (1997).

[5] V. Malka, S. Fritzler, E. Lefebvre, M.-M. Aleonard, F. Burgy, J.-P. Chambaret, J.-F. Chemin, K. Krushelnick, G. Malka, S.P.D. Mangles, Z. Najmudin, M. Pittman, J.-P. Rousseau, J.-N. Scheurer, B. Walton, and A.E. Dangor, Science 298, 1596 (2002).

[6] M.I.K. Santala, Z. Najmudin, E.L. Clark, M. Tatarakis, K. Krushelnick, A.E. Dangor, V. Malka, J. Faure, R. Allott, and R.J. Clarke, Phys. Rev. Lett. 86, 1227 (2001).

[7] S.P.D. Mangles, C.D. Murphy, Z. Najmudin, A.G.R. Thomas, J.L. Collier, A.E. Dangor, E.J. Divall, P.S. Foster, J.G. Gallacher, C.J. Hooker, D.A. Jaroszynski, A.J. Langley, W.B. Mori, P.A. Norreys, F.S. Tsung, R. Viskup, B.R. Walton, and K. Krushelnick, Nature 431, 535 (2004).

[8] J. Faure, Y. Glinec, A. Pukhov, S. Kiselev, S. Gordienko, E. Lefebvre, J.-P. Rousseau, F. Burgy, and V. Malka, Nature 431, 541 (2004); C.G.R. Geddes, Cs. Toth, J. van Tilborg, E. Esarey, C.B. Schroeder, D. Bruhwiler, C. Nieter, J. Cary, and W.P. Leemans, Nature 431, 538 (2004); A. Pukhov and J. Meyer-ter-Vehn, Appl. Phys. B 74, 355 (2002).

[9] S.P.D. Mangles, B.R. Walton, M. Tzoufras, Z. Najmudin, R.J. Clarke, A.E. Dangor, R.G. Evans, S. Fritzler, A. Gopal, C. Hernandez-Gomez, W.B. Mori, W. Rozmus, M. Tatarakis, A.G.R. Thomas, F.S. Tsung, M.S. Wei, and K. Krushelnick, Phys. Rev. Lett. 94, 245001 (2005).

[10] A.G.R. Thomas, Z. Najmudin, S.P.D. Mangles, C.D. Murphy, A.E. Dangor, C. Kamperidis, K.L. Lancaster, W.B. 
Mori, P.A. Norreys, W. Rozmus, and K. Krushelnick, Phys. Rev. Lett. 98, 095004 (2007).

[11] S.P.D. Mangles, A.G.R. Thomas, M.C. Kaluza, O. Lundh, F. Lindau, A. Persson, Z. Najmudin, C.-G. Wahlström, C.D. Murphy, C. Kamperidis, K.L. Lancaster, E. Divall, and K. Krushelnick, Plasma Phys. Control. Fusion 48, B83 (2006).

[12] B.R. Walton, S.P.D. Mangles, Z. Najmudin, M. Tatarakis, M.S. Wei, A. Gopal, C. Marle, A.E. Dangor, K. Krushelnick, S. Fritzler, V. Malka, R.J. Clarke, and C. Hernandez-Gomez, Phys. Plasmas 13, 113103 (2006).

[13] S.P.D. Mangles, A.G.R. Thomas, M.C. Kaluza, O. Lundh, F. Lindau, A. Persson, F.S. Tsung, Z. Najmudin, W.B. Mori, C.-G. Wahlström, and K. Krushelnick, Phys. Rev. Lett. 96, 215001 (2006).

[14] C.D. Murphy, R. Trines, J. Vieira, A.J.W. Reitsma, R. Bingham, J.L. Collier, E.J. Divall, P.S. Foster, C.J. Hooker, A.J. Langley, P.A. Norreys, R.A. Fonseca, F. Fiuza, L.O. Silva, J.T. Mendonça, W.B. Mori, J.G. Gallacher, R. Viskup, D.A. Jaroszynski, S.P.D. Mangles, A.G.R. Thomas, K. Krushelnick, and Z. Najmudin, Phys. Plasmas 13, 033108 (2006).

[15] S.P.D. Mangles, K. Krushelnick, Z. Najmudin, M.S. Wei, B. Walton, A. Gopal, A.E. Dangor, S. Fritzler, C.D. Murphy, A.G.R. Thomas, W.B. Mori, J. Gallacher, D. Jaroszynski, P.A. Norreys, and R. Viskup, Phil. Trans. R. Soc. A 364, 663 (2006).

[16] K. Krushelnick, Z. Najmudin, S.P.D. Mangles, A.G.R. Thomas, M.S. Wei, B. Walton, A. Gopal, E.L. Clark, A.E. Dangor, S. Fritzler, C.D. Murphy, P.A. Norreys, W.B. Mori, J. Gallacher, D. Jaroszynski, and R. Viskup, Phys. Plasmas 12, 056711 (2005).

[17] Z. Najmudin, K. Krushelnick, M. Tatarakis, E.L. Clark, C.N. Danson, V. Malka, D. Neely, M.I.K. Santala, and A.E. Dangor, Phys. Plasmas 10, 438 (2003).

[18] Z. Najmudin, K. Krushelnick, E.L. Clark, S.P.D. Mangles, B. Walton, A.E. Dangor, S. Fritzler, V. Malka, E. Lefebvre, D. Gordon, F.S. Tsung, and C. Joshi, Phys. Plasmas 10, 2071 (2003).

[19] Z. Najmudin, K. Krushelnick, E.L. Clark, D.J. Colling, M. Tatarakis, A. Modena, A.E. Dangor, J. Faure, V. Malka, D. Gordon, and C. Joshi, J. Modern Opt. 50, 673 (2003).

[20] F. Amiranoff, S. Baton, D. Bernard, B. Cros, D. Descamps, F. Dorchies, F. Jacquet, V. Malka, J.R. Marquès, G. Matthieussent, P. Miné, A. Modena, P. Mora, J. Morillo, and Z. Najmudin, Phys. Rev. Lett. 81, 995 (1998).

[21] K. Krushelnick and Z. Najmudin, Phys. World 19 (2), 24 (2006).

[22] W.P. Leemans, B. Nagler, A.J. Gonsalves, Cs. Tóth, K. Nakamura, C.G.R. Geddes, E. Esarey, C.B. Schroeder, and S.M. Hooker, Nature Phys. 2, 696 (2006).

[23] I. Blumenfeld, C.E. Clayton, F.-J. Decker, M.J. Hogan, C. Huang, R. Ischebeck, R. Iverson, C. Joshi, T. Katsouleas, N. Kirby, W. Lu, K.A. Marsh, W.B. Mori, P. Muggli, E. Oz, R.H. Siemann, D. Walz, and M. Zhou, Nature 445, 741 (2007).

[24] M. Everett, A. Lal, D. Gordon, C.E. Clayton, K.A. Marsh, and C. Joshi, Nature 368, 527 (1994).

[25] B. Walton, Z. Najmudin, M.S. Wei, C. Marle, R.J. Kingham, K. Krushelnick, A.E. Dangor, R.J. Clarke, M.J. Poulter, C. Hernandez-Gomez, S. Hawkes, D. Neely, J.L. Collier, C.N. Danson, S. Fritzler, and V. Malka, Opt. Lett. 27, 2203 (2002).
[26] B. Walton, Z. Najmudin, M.S. Wei, C. Marle, R.J. Kingham, K. Krushelnick, A.E. Dangor, R.J. Clarke, M.J. Poulter, C. Hernandez-Gomez, S. Hawkes, D. Neely, J.L. Collier, C.N. Danson, S. Fritzler, and V. Malka, Phys. Plasmas 13, 013103 (2006).

[27] C. Gahn, G.D. Tsakiris, A. Pukhov, J. Meyer-ter-Vehn, G. Pretzler, P. Thirolf, D. Habs, and K.J. Witte, Phys. Rev. Lett. 83, 4772 (1999).

[28] J. Meyer-ter-Vehn and Z.M. Sheng, Phys. Plasmas 6, 641 (1999).

[29] R.A. Fonseca, L.O. Silva, F.S. Tsung, V.K. Decyk, W. Lu, C. Ren, W.B. Mori, S. Deng, S. Lee, T. Katsouleas, and J.C. Adam, in: Proc. of the Int. Conf. Comp. Sci., Amsterdam, Netherlands, 21-24 April 2002 (ICCS 2002), Lecture Notes in Computer Science 2331, 342 (2002).

[30] J. Faure, Y. Glinec, J.J. Santos, F. Ewald, J.-P. Rousseau, S. Kiselev, A. Pukhov, T. Hosokai, and V. Malka, Phys. Rev. Lett. 95, 205003 (2005).

[31] W. Lu, C. Huang, M. Zhou, W.B. Mori, and T. Katsouleas, Phys. Rev. Lett. 96, 165002 (2006).

[32] S.V. Bulanov, F. Pegoraro, A.M. Pukhov, and A.S. Sakharov, Phys. Rev. Lett. 78, 4205 (1997).

[33] A.G.R. Thomas, S.P.D. Mangles, Z. Najmudin, M.C. Kaluza, C.D. Murphy, and K. Krushelnick, Phys. Rev. Lett. 98, 054802 (2007).

[34] S.J. Gitomer, R.D. Jones, F. Begay, A.W. Ehler, J.F. Kephart, and R. Kristal, Phys. Fluids 29, 2679 (1984).

[35] F. Begay and D.W. Forslund, Phys. Fluids 25, 1675 (1982); T.H. Tan, G.H. McCall, and A.H. Williams, Phys. Fluids 27, 296 (1984); A.H. Ehler, J. Appl. Phys. 46, 2464 (1975).

[36] L.M. Wickens, J.E. Allen, and P.T. Rumsby, Phys. Rev. Lett. 41, 243 (1978).

[37] A.P. Fews, P.A. Norreys, F.N. Beg, A.R. Bell, A.E. Dangor, C.N. Danson, P. Lee, and S.J. Rose, Phys. Rev. Lett. 73, 1801 (1994).

[38] E.L. Clark, K. Krushelnick, M. Zepf, F.N. Beg, M. Tatarakis, A. Machacek, M.I.K. Santala, I. Watts, P.A. Norreys, and A.E. Dangor, Phys. Rev. Lett. 85, 1654 (2000).

[39] K.W.D. Ledingham, I. Spencer, T. McCanny, R.P. Singhal, M.I.K. Santala, E. Clark, I. Watts, F.N. Beg, M. Zepf, K. Krushelnick, M. Tatarakis, A.E. Dangor, P.A. Norreys, R. Allott, D. Neely, R.J. Clark, A.C. Machacek, J.S. Wark, A.J. Cresswell, D.C.W. Sanderson, and J. Magill, Phys. Rev. Lett. 84, 899 (2000); M.I.K. Santala, M. Zepf, I. Watts, F.N. Beg, E. Clark, M. Tatarakis, K. Krushelnick, A.E. Dangor, T. McCanny, I. Spencer, R.P. Singhal, K.W.D. Ledingham, S.C. Wilks, A.C. Machacek, J.S. Wark, R. Allott, R.J. Clarke, and P.A. Norreys, Phys. Rev. Lett. 84, 1459 (2000).

[40] P.A. Norreys, A.P. Fews, F.N. Beg, A.R. Bell, A.E. Dangor, P. Lee, M.B. Nelson, H. Schmidt, M. Tatarakis, and M.D. Cable, Plasma Phys. Control. Fusion 40, 175 (1998).

[41] L. Disdier, J.-P. Garçonnet, G. Malka, and J.-L. Miquel, Phys. Rev. Lett. 82, 1454 (1999).

[42] L.O. Silva, M. Marti, J.R. Davies, R.A. Fonseca, C. Ren, F.S. Tsung, and W.B. Mori, Phys. Rev. Lett. 92, 015002 (2004).

[43] E.L. Clark, K. Krushelnick, J.R. Davies, M. Zepf, M. Tatarakis, F.N. Beg, A. Machacek, P.A. Norreys, M.I.K. Santala, I. Watts, and A.E. Dangor, Phys. Rev. Lett. 84, 670 (2000). 
[44] M.I K. Santala, M. Zepf, F.N. Beg, E.L. Clark, A.E. Dangor, K. Krushelnick, M. Tatarakis, I. Watts, K.W.D. Ledingham, T. McCanny, I. Spencer, A.C. Machacek, R. Allott, R.J. Clarke, and P.A. Norreys, Appl. Phys. Lett. 78, 19 (2001).

[45] M. Zepf, E.L. Clark, F.N. Beg, R.J. Clarke, A.E. Dangor, A. Gopal, K. Krushelnick, P.A. Norreys, M. Tatarakis, U. Wagner, and M.S. Wei, Phys. Rev. Lett. 90, 064801 (2003); M. Zepf, E.L. Clark, K. Krushelnick, F.N. Beg, C. Escoda, A.E. Dangor, M.I.K. Santala, M. Tatarakis, I.F. Watts, P.A. Norreys, R.J. Clarke, J.R. Davies, M.A. Sinclair, R.D. Edwards, T.J. Goldsack, I. Spencer, and K.W.D. Ledingham, Phys. Plasmas 8, 2323 (2001).

[46] R.A. Snavely, M.H. Key, S.P. Hatchett, T.E. Cowan, M. Roth, T.W. Phillips, M.A. Stoyer, E.A. Henry, T.C. Sangster, M.S. Singh, S.C. Wilks, A. MacKinnon, A. Offenberger, D.M. Pennington, K. Yasuike, A.B. Langdon, B.F. Lasinski, J. Johnson, M.D. Perry, and E.M. Campbell, Phys. Rev. Lett. 85, 2945 (2000).

[47] H. Schwoerer, S. Pfotenhauer, O. Jäckel, K.-U. Amthor, B. Liesfeld, W. Ziegler, R. Sauerbrey, K.W.D. Ledingham, and T. Esirkepov, Nature 439, 445 (2006); B.M. Hegelich, B.J. Albright, J. Cobble, K. Flippo, S. Letzring, M. Paffett, H. Ruhl, J. Schreiber, R.K. Schulze, and J.C. Fernández, Nature 439, 441 (2006).

[48] T.E. Cowan, J. Fuchs, H. Ruhl, A. Kemp, P. Audebert, M. Roth, R. Stephens, I. Barton, A. Blazevic, E. Brambrink, J. Cobble, J. Fernández, J.-C. Gauthier, M. Geissel, M. Hegelich, J. Kaae, S. Karsch, G.P. Le Sage, S. Letzring, M. Manclossi, S. Meyroneinc, A. Newkirk, H. Pépin, and N. Renard-LeGalloudec, Phys. Rev. Lett. 92, 204801 (2004).

[49] M. Hegelich, S. Karsch, G. Pretzler, D. Habs, K. Witte, W. Guenther, M. Allen, A. Blazevic, J. Fuchs, J.C. Gauthier, M. Geissel, P. Audebert, T. Cowan, and M. Roth, Phys. Rev. Lett. 89, 085002 (2002).
[50] L. Willingale, S.P.D. Mangles, P.M. Nilson, R.J. Clarke, A.E. Dangor, M.C. Kaluza, S. Karsch, K.L. Lancaster, W.B. Mori, Z. Najmudin, J. Schreiber, A.G.R. Thomas, M.S. Wei, and K. Krushelnick, Phys. Rev. Lett. 96, 245002 (2006).

[51] K. Krushelnick, E.L. Clark, Z. Najmudin, M. Salvati, M.I.K. Santala, M. Tatarakis, A.E. Dangor, V. Malka, D. Neely, R. Allott, and C. Danson, Phys. Rev. Lett. 83, 737 (1999); M.S. Wei, S.P.D. Mangles, Z. Najmudin, B. Walton, A. Gopal, M. Tatarakis, A.E. Dangor, E.L. Clark, R.G. Evans, S. Fritzler, R.J. Clarke, C. Hernandez-Gomez, D. Neely, W. Mori, M. Tzoufras, and K. Krushelnick, Phys. Rev. Lett. 93, 155003 (2004).

[52] S. Fritzler, Z. Najmudin, V. Malka, K. Krushelnick, C. Marle, B. Walton, M.S. Wei, R.J. Clarke, and A.E. Dangor, Phys. Rev. Lett. 89, 165004 (2002).

[53] R.D. Edwards, M.A. Sinclair, T.J. Goldsack, K. Krushelnick, F.N. Beg, E.L. Clark, A.E. Dangor, Z. Najmudin, M. Tatarakis, B. Walton, M. Zepf, K.W.D. Ledingham, I. Spencer, P.A. Norreys, R.J. Clarke, R. Kodama, Y. Toyama, and M. Tampo, Appl. Phys. Lett. 80, 2129 (2002).

[54] K. Krushelnick, E.L. Clark, F.N. Beg, A.E. Dangor, Z. Najmudin, P.A. Norreys, M. Wei, and M. Zepf, Plasma Phys. Control. Fusion 47, B451 (2005).

[55] P. McKenna, K.W.D. Ledingham, T. McCanny, R.P. Singhal, I. Spencer, M.I.K. Santala, F.N. Beg, K. Krushelnick, M. Tatarakis, M.S. Wei, E.L. Clark, R.J. Clarke, K.L. Lancaster, P.A. Norreys, K. Spohr, R. Chapman, and M. Zepf, Phys. Rev. Lett. 91, 075006 (2003).

[56] M. Borghesi, A. Schiavi, D.H. Campbell, M.G. Haines, O. Willi, A.J. MacKinnon, L.A. Gizzi, M. Galimberti, R.J. Clarke, and H. Ruhl, Plasma Phys. Control. Fusion 43, A267 (2001); M. Borghesi, S. Bulanov, D.H. Campbell, R.J. Clarke, T.Zh. Esirkepov, M. Galimberti, L.A. Gizzi, A.J. MacKinnon, N.M. Naumova, F. Pegoraro, H. Ruhl, A. Schiavi, and O. Willi, Phys. Rev. Lett. 88, 135002 (2002). 